\title{
Production and Separation of Radioactive lon Beams
}

\author{
Marco Mazzocco ${ }^{1,2, \star}$ \\ ${ }^{1}$ Dipartimento di Fisica e Astronomia, Universitá di Padova, via F. Marzolo 8, I-35131 Padova, Italy \\ 2 INFN - Sezione di Padova, via F. Marzolo 8, I-35131 Padova, Italy
}

\begin{abstract}
Radioactive nuclei play an important role in many astrophysical scenarios, from the Big-Bang Nucleo-synthesis to the standard solar model, from quiescent burning to the most explosive events that can occur in our universe. A huge effort has been made for more than thirty years to construct facilities able to deliver beams of radioactive nuclei with increasing intensity and better quality. This contribution revises the different mechanisms and the separation techniques employed for the production of Radioactive Ion Beams.
\end{abstract}

\section{Introduction}

If we a take even a quick look to the nuclide chart, we can immediately realize the reason why nowadays Nuclear Physics and Nuclear Astrophysics are deeply interested in studying the properties and the reactions induced by radioactive nuclei. In the entire spectrum of combinations between protons and neutrons, we find only 198 stable nuclei and 86 metastable nuclei, i.e. nuclei with half lives longer than age of the earth. So far, 3302 nuclides have been discovered (December 2018 [1]) and more than 6000 nuclei are postulated to exist. While studying exotic nuclei far from the valley of $\beta$-stability, we might discover new decay modes, observe the evolution of the shell closures and probe the limits of existence of nuclei.

From the point of view of Nuclear Astrophysics, radioactive nuclei are involved in many process of astrophysical interest, such as, for instance, the Big-Bang Nucleosynthesis, novae and supernovae explosions, $\mathrm{x}$-ray bursts and so on. Even in our Sun, the radioactive nuclei ${ }^{7} \mathrm{Be},{ }^{8} \mathrm{Be}$ and ${ }^{8} \mathrm{~B}$ are populated in the so-called ppII and ppIII branches of the standard solar model. In addition, about $0.8 \%$ of the energy generated in the Sun comes from the CNO cycle, which also includes a few radioactive nuclei.

As beautifully described by M. Thoennessen in his essay [2], our knowledge of radioactive nuclei followed essentially the development of particle accelerators and the history of the human being over the last 125 years. At first only the products of the radioactive decays of the primordial radionuclides present on earth were investigated, then it came the age of mass spectroscopy and of the first accelerators. After the second World War, the development of nuclear reactors determined a huge boost in the investigation of nuclei far from stability, especially fission fragments. With the availability of more powerful heavy-ion accelerators we had the possibility of producing nuclei via fusion-evaporation

\footnotetext{
^e-mail: marco.mazzocco@pd.infn.it
} 
reactions. The increasing beam energy at our disposal then opened up the radioactive ion beam production via projectile fragmentation and, eventually, we had the final boom determined by the advent of second generation Radioactive Ion Beam (RIB) facilities.

While studying exotic nuclei far from the valley of stability we have somewhat to fight against the weak interaction, which slides radioactive nuclei along isobaric chains towards the bottom of the valley. In addition, the half-lives for the $\beta$ decay become shorter and shorter while moving towards the neutron and proton drip-lines, making the investigation of very exotic more and more challenging.

The global production scheme for the RIBs should, therefore:

- be fast, because of the very short half-lives of the nuclear species under investigation;

- warranty the largest possible production cross section. This issue is achieved by optimizing the production reaction after a careful choice of projectile-target combination, bombarding energy, primary beam intensity, power deposition into the primary target, ...

- be efficient, especially because of the, in most cases, very low production yields;

- be selective, since, very frequently, contaminants might be several orders of magnitude more abundant than the radioisotopes we are interested in.

\section{RIB Production Mechanisms}

There exist four main mechanisms for the production of RIBs: projectile fragmentation (mostly for neutron-deficient nuclei), projectile fission (essentially for neutron-rich nuclei), nuclear fusion (for proton-rich nuclei and for the synthesis of superheavy nuclei) and two-body processes in inverse kinematics (basically for light RIBs). We will briefly describe these four processes.

\subsection{Projectile Fragmentation}

The projectile fragmentation is a peripheral collision with a target nucleus, leading to the removal of a few projectile nucleons. To be effective the bombarding energy must be higher than $40 \mathrm{MeV} / \mathrm{u}$. Above this threshold energy, the cross section for fragmentation is, in a first approximation, practically constant. To reach this energy, the RIB facility must be equipped with at least a cyclotron, or even a synchrotron, as driver accelerator. The advantage of increasing the beam energy is related to the fact the residual nuclei produced by the fragmentation process are emitted in narrower cones around the primary beam direction and the lay-out of spectrometers with a smaller opening, better optical transmission and lower cost can be considered.

\subsection{Projectile Fission}

The second mechanism we present is the projectile fission. The key ingredient in this case is, of course, a fissile projectile, typically ${ }^{238} \mathrm{U}$. Fission fragments are emitted with kinetic energies of around 1 $\mathrm{MeV} / \mathrm{u}$ (in the center of mass reference frame), which translates into emission cones around the primary beam axis with significantly larger openings than in fragmentation processes. Therefore, in this case, the increase of the projectile energy is much more relevant for an efficient production of secondary beams of exotic species far from stability. 


\subsection{Nuclear Fusion}

Technically speaking, nuclear fusion is not a mechanism employed so far for the RIB production. It is rather used for spectroscopy studies and for the synthesis of super-heavy nuclei and, only in that respect, can be regarded as a secondary beam production technique. Nuclear fusion makes use of the lower velocity of fusion-evaporation residues with respect to projectile velocity to operate a selection by means of ion-optical elements. The separation efficiency of this technique is quite limited since the residues are produced with broad angle, velocity and charge state distributions, as a direct consequence of the fact that the beam energy required by this process to be effective is pretty low (around $5 \mathrm{MeV} / \mathrm{u}$ ).

\subsection{Two-Body Reactions in Inverse Kinematics}

The last mechanism we consider is when a heavy-ion beam impinges on a light target and induces a two-body reaction, such as (p.n), (d,n) or $\left({ }^{3} \mathrm{He}, \mathrm{n}\right)$. Linear momentum conservation implies that the radioactive nuclei are emitted in rather narrow cones around the primary beam direction and the high cross sections and the limited number of nuclear species produced made this process quite efficient, especially for light RIBs. A few tricks might be employed in order to optimize the separation quality. For instance, when possible, the choice of a nuclear reaction with negative $Q_{v a l u e}$ and with a forwardpeaked differential cross section is a net advantage.

\section{RIB Separation Techniques}

After the production reaction, RIBs are generally separated and selected by mean of two complementary techniques: In-Flight separation and Isotope Separation On-Line (ISOL). We will present the main features of both methods.

\subsection{In-Flight}

This techniques exploits the kinematic (forward) focusing, due to the linear momentum conservation, at small angles around the primary beam direction of the reaction products and allows for a fast separation of the exotic species. The separation is independent from the chemical properties of the produced isotopes and is performed by means of a proper combinations of electromagnetic fields and atomic interaction.

Within a region where a magnetic field $B$, orthogonal to beam direction, is present, particles are deflected according to their mass $m$, velocity $v$ and charge state $q$ along trajectories with radius of curvature $\rho=\frac{m v}{q B}$. Being, typically, the magnetic field constant, nuclides are essentially selected according to their magnetic rigidity $B \rho=\frac{m v}{q}$, which depends only on particle observables. The separation quality can be optimized by displacing at suitable locations along the beam lines properly shaped degraders, where the produced nuclear species will undergo atomic interaction. Being the energy loss quite selective in terms of the atomic number of the incoming particles, we might exploit this feature to get rid of unwanted species and improve the purity of the radioisotopes of interest.

Moreover, since the RIBs retain a large fraction of the initial projectile velocity, in-flight RIBs do not need to be re-accelerated and the lay-out and construction of an additional, rather expensive, post-accelerator. This technique, however, presents also some drawbacks, such as, a generally poor ion optical beam quality, in terms of high longitudinal and transverse emittance, and large beam spots on target. In addition, the production target should be able to withstand the large power deposition of the primary beam and the transport magnets, especially the closest ones to the production area, must be designed to function also in high radiation environment. 
A detailed description of the in-flight technique can be found in the review article [3]. Facilities like the FRS [4] (GSI, Germanay), RIPS [5] and Big-RIPS [6] (RIKEN, Japan), for instance, make use of the in-flight technique for the production of Radioactive Ion Beams, especially by means of the projectile fragmentation and projectile fission reaction mechanisms. In the low-energy regime, the facilities CRIB (CNS and RIKEN, Japan) [7], MARS [8] (USA), TwinSol [9] (Notre Dame, USA), ANL [10] (USA), RIBRAS [11] (Brazil), SOLEROO [12] (Australia) and EXOTIC [13] (Italy) produce secondary beams employing two-body processes.

\subsection{ISOL}

In the case of the ISOL technique, the exotic species are produced within a thick target, which, depending on the layout of the facility, stops completely, or nearly completely, the primary beam. The target station is generally kept at very high temperature, in such a way that the thermalized exotic nuclei can diffuse within the target material and then effuse towards the ion source. These two processes strongly depend on the chemical properties of the produced species, which, at this stage of the production, still have very low velocity and are uncharged. To be accelerated, particles need to be ionized, therefore the target-ion-source must be equipped with an ionization mechanism, based either on electron impact ionization, surface ionization or laser resonance photo-ionization. These three mechanisms have rather different efficiency and selectivity, thus the facility is quite frequently a high resolution mass separator with large dispersion is added to remove undesired nuclei and further purity the secondary beams.

ISOL beams needs to be re-accelerated in order to match the energy range of interest for Nuclear Physics studies and to enhance the performances of the post accelerator, the charge state of the RIBs has to increased by means of a proper device, called charge breeder, which shares a certain number of similarities with a positive ion source. Being re-accelerated, ISOL beams have very good ion optical properties, very small emittance and their kinetic energy might be varied with the entire spectrum allowed by the particle accelerator.

More details on the ISOL technique are described in the review article [14]. Facilities like the REX-ISOLDE [15] (CERN, Switzerland) and SPIRAL [16] (GANIL, France), for example, employ this secondary beam production technique.

\section{Outlook}

The nuclear reaction mechanisms and the separation techniques employed for the production of RIBs have been reviewed in this contribution. The choice of the most useful mechanism and technique is also based on the implementations and the experimental setup available in the laboratory where an experiment is carried out.

When a few degrees of freedom are present, we rather choose IF secondary beams when the halflife of the RIB under investigation is shorter than a few hundred milliseconds. In case of IF RIBs, the main limitation on time arises from the transit time through the electromagnetic separator, typically of the order of tens of microseconds (but clearly related to the physical length of the separator). When we need a secondary beam of very high energy, i.e. larger than $100 \mathrm{MeV} / \mathrm{u}$, we also rather prefer IF beams, since the re-acceleration of an energetic secondary beam would require the construction of a new dedicated particle accelerator.

On the contrary, when the beam optical qualities, in terms of energy resolution, beam emittance, beam spot on target, are more relevant, if available on the market, the best choice would be to use a post-accelerated ISOL beam, which would allow also a quite flexible variation of the beam energy in an extremely wide range. 


\section{References}

[1] https://people.nscl.msu.edu/ thoennes/isotopes/

[2] M. Thoennessen et al., Nature 473, 25 (2011)

[3] D.J. Morrissey and B.M. Sherill, Lect. Notes Phys. 651, 113 (2004)

[4] H. Geissel et al., Nucl. Instrum. Meth. B 70, 286 (1992)

[5] T. Kubo et al, Nucl. Instrum. Meth. B 70, 309 (1992).

[6] T. Kubo et al, Nucl. Instrum. Meth. B 204, 97 (2003).

[7] Y. Yanagisawa et al., Nucl. Instrum. Meth. A 539, 74 (2005) and Y. Yamaguchi et al., Nucl. Instrum. Meth. A 589, 150 (2008)

[8] R.E. Tribble et al., Nucl. Instrum. Meth. A 289, 441 (1989)

[9] F.D. Becchetti et al., Nucl. Instrum. Meth. A 505, 377 (2003)

[10] B. Harss et al., Rev. Sci. Instrum. 71, 380 (2000)

[11] R. Lichtenthaler et al., Eur. Phys. J. A 25, s733 (2005)

[12] R. Rafiei et al., Nucl. Instrum. Meth. A 631, 12 (2011)

[13] F. Farinon et al., Nucl. Instrum. Meth. B 266, 4097 (2008)

[14] P. Van Duppen, Lect. Notes Phys. 700, 37 (2006)

[15] http://isolde.web.cern.ch/

[16] A.C.C. Villari et al., Nucl. Instr. Meth. B 204, 31 (2003) 